Rev Soc Esp Dolor

2015; 22(2): 91-92

\section{Réplica: Objeciones que invalidan las conclusiones del estudio de efectividad, tolerabilidad y calidad de vida de tapentadol LP vs. oxicodona/naloxona LP}

\section{Sr. Director:}

En respuesta a la carta al editor anterior, se detalla a continuación punto por punto por qué el diseño y el desarrollo del estudio efectividad, tolerabilidad y calidad de vida de tapentadol LP vs. oxicodona/naloxona $L P$ son apropiados y apoyan el perfil favorable de eficacia y tolerabilidad de tapentadol LP. Este documento precede a la publicación del manuscrito completo del estudio que se llevará a cabo en 2015 y se refiere a las presentaciones realizadas durante el Congreso PainWEEK 2014 (1-3).

Nuestro estudio fue diseñado para estudiar la hipótesis de no-inferioridad (seguida de la de superioridad) de tapentadol LP versus oxicodona/naloxona LP $(\mathrm{OXN})$ en un análisis estadístico pre-planeado en 2 etapas para 2 variables primarias. La hipótesis del estudio se basó en la justificación de que la eficacia de OXN es comparable a la de oxicodona LC (OXY) (4) y que la diferencia de tolerabilidad de OXN versus OXY se debe al efecto (no sistémico) de la naloxona en el estreñimiento inducido por opioides.

1. Diseño abierto vs. diseño doble ciego: el diseño abierto para este estudio se consideró adecuado. La evidencia derivada de los ensayos clínicos aleatorizados y controlados (RCT) para evaluar la efectividad (práctica habitual/estudios pragmáticos) cumplen el nivel de evidencia Ib de acuerdo con los estándares de la medicina basada en la evidencia así como de las Revisiones Cochrane. Los estudios abiertos son aceptados por las Agencias Reguladoras y por las Agencias de Evaluación Tecnológica de la Salud (AETS) con el fin de completar la evidencia obtenida en los estudios aleatorizados controlados doble ciego y mejorar las limitaciones asociadas a los mismos (p. ej., poblaciones seleccionadas, diseños poco adaptados a la práctica clínica habitual). Además, los ensayos clínicos aleatorizados y controlados diseñados para evaluar la seguridad/tolerabilidad habitualmente utilizan diseños abiertos en línea con las directrices de las autoridades reguladoras. En este contexto, los resultados de nuestro estudio son consistentes con los resultados de tres ensayos clínicos pivotales aleatorizados y controlados con placebo en dolor por artrosis de rodilla y dolor lumbar; estos resultados además han sido apoyados por análisis conjuntos de datos pre-planeados y actualmente publicados donde se ha demostrado la superio- ridad de tapentadol LP sobre OXY para la variable primaria de eficacia, parámetros de calidad de vida validados y tolerabilidad gastrointestinal (GI) $(5,6)$.

2. Brazo de rescate: en primer lugar es importante aclarar que la justificación del brazo de rescate de este estudio no proviene de los resultados del ensayo clínico de rotación (7) donde se demostró la mejoría en tolerabilidad/efectividad cuando se rotaba de un opioide potente a tapentadol LP. Por otro lado, el brazo de rescate tiene una justificación ética y científica directa sin introducir ningún sesgo relevante en el estudio. Tapentadol LP ofrece una eficacia mejorada en comparación con OXY (principio activo de OXN) basado en sus dos mecanismos de acción (MOR-NRI), tal y como se ha demostrado en ensayos clínicos aleatorizados doble-ciego con una población amplia de pacientes. Además la superioridad de tapentadol LP versus OXY con respecto a la tolerabilidad GI incluye otros efectos adversos, no sólo el estreñimiento (5), donde OXN puede ser favorable versus OXY. Se planeó el brazo de rescate con la intención de dar a los pacientes la opción de alcanzar un alivio de dolor adecuado con una tolerabilidad aceptable, una situación que se puede considerar reflejo de la práctica clínica habitual; en contra, era improbable que el cambio de tapentadol LP a OXN (basado en la evidencia) aportara beneficios adicionales. Los resultados del estudio indican que el brazo de rescate no influyó en la tasa de discontinuación: la tasa de abandono del tratamiento con OXN en este estudio $(62,5 \%)$ fue comparable a la de OXY $(61,7 \%)$ en ensayos clínicos fase III aleatorizados, controlados y doble ciego (5). En resumen, se puede considerar que los resultados de este estudio son consistentes con los estudios previos aleatorizados, controlados y doble-ciego sin brazo de rescate.

3.1. Esquema de titulación de la dosis: la pauta de titulación usada para en este estudio se puede considerar equitativa y adecuada en el contexto de este tipo de ensayo y objetivos del mismo. Este esquema permitía un ratio equianalgésico para el incremento de dosis, evitando el sesgo de infradosificación de uno de los fármacos en el caso de titulación de dosis de manera desigual. Adicionalmente, es importante destacar que una titulación más lenta (cada 3 días) cubre las necesidades de una población sensible no pretratada con opioides, evitando los sesgos de una titulación más rápida de uno de los fármacos. Además, las pautas de titulación uti- 
lizadas para OXN pueden variar; generalmente no está permitido OXN 5/2,5 g como dosis de inicio y ascensos de dosis con OXN 10/5 mg dos veces al día están en línea con la Información de Prescripción de la FDA. Un intervalo de titulación más prolongado es bastante común como se muestra en el ensayo clínico aleatorizado y controlado de OXN en dolor crónico lumbar utilizando incrementos de 10/5 mg dos veces al día, una vez a la semana (8).

3.2. Respuesta al criterio para la fase de titulación: para entrar en la fase de mantenimiento del estudio existían dos criterios: el primero requería una intensidad de dolor $\leq 4$ (en la escala validada EVN-3) con una tolerabilidad aceptable al final del periodo de titulación; el segundo criterio (mencionado en la Carta al Director) se refería a ofrecer una opción a los pacientes de continuar el tratamiento si el dolor se había reducido a $\leq 5$ con la dosis más alta tolerada. La objeción en la Carta al Director sobre una reducción de un punto en la escala EVN-3 era un criterio inicial teórico de respuesta (apoyado por los criterios internacionales IMMPACT para una diferencia clínica mínimamente importante). Es importante destacar que todos los pacientes de cada grupo excepto uno obtuvieron un alivio de dolor $\geq 2$ puntos al final de la titulación (población por protocolo).

4. Pre-tratamiento IRN (inhibición de la recaptación de noradrenalina): los antidepresivos (incluidos los IRN) se permitían sólo como medicación concomitante para pacientes con depresión y a dosis estables, al igual que en otros ensayos clínicos (incluidos estudios de OXN) (8). Cualquier co-analgésico (para el dolor en estudio) tenía que ser "lavado" antes de la aleatorización. Ninguno de los pacientes del estudio recibió co-analgésicos previos con mecanismo de acción inhibidor de la recaptación de noradrenalina. El concepto teórico de que pacientes que continuasen el tratamiento con IRN fuesen pacientes "no naïves" a los efectos secundarios de los IRNS no está avalado científicamente (en la práctica clínica existe un impacto mínimo de las reacciones adversas o de la no tolerancia a los efectos adversos notificadas y derivadas de la inhibición de la recaptación de noradrenalina). El tratamiento concomitante con IRN puede incluso favorecer a OXN, puesto que le añade el efecto sinérgico de un opioide y un inhibidor de la recaptación de noradrenalina en el alivio del dolor.

5. Evaluación de la función intestinal: en base a numerosos programas clínicos con opioides, se conoce que la escala validada PAC-SYM cubre la función intestinal en general y, utilizada como variable primaria, puede ser menos sensible a la notificación de forma espontánea del estreñimiento como efecto adverso. En nuestro estudio se ha demostrado la no-inferioridad de tapentadol LP versus OXN para la variable co-primaria. Además, el estudio presenta el sesgo en el análisis del PAC-SYM a favor de OXN puesto que es el grupo con una tasa de discontinuación de tratamiento más elevada en la fase de titulación. La puntuación PAC-SYM se evaluaba solamente en la visita basal y en la visita final o de discontinuación del estudio; por tanto, no fue posible realizar la imputación de los valores perdidos por media aritmética por el último valor notificado con una función intestinal normal en ningún grupo de tratamiento (p. ej., en la visita basal), y se asume un mejor resultado del actualmente obtenido. En este contexto, se observa con tapentadol LP una incidencia de estreñimiento espontáneamente notificado por el paciente $40 \%$ menor (estadísticamente significativo) que con $\mathrm{OXN}(2)$.

\section{R. Baron ${ }^{1}$ e I. Steigerwald ${ }^{2}$}

${ }^{1}$ División de Investigación y Terapia del Dolor. Clínica Universitaria Schleswig-Holstein. Kiel, Alemania. ${ }^{2}$ Departamento Médico Europa \& Australia. Grünenthal GmbH. Alemania

\section{BIBLIOGRAFÍA}

1. Baron R, et al. Effectiveness of tapentadol prolonged release (PR) versus oxycodone/naloxone PR for severe chronic low back pain with a neuropathic pain component. PAIN Week, Las Vegas, USA 2014. Abstract 121. Available at: http://conference.painweek.org/media/mediafile_attachments/04/724painweek2014acceptedabstracts.pdf.

2. Binder A, et al. Safety and tolerability of tapentadol prolonged release (PR) versus oxycodone/naloxone PR for severe chronic low back pain with a neuropathic pain component. PAIN Week Las Vegas, USA 2014. Abstract 122. Available at: http://conference.painweek.org/media/mediafile_ attachments/04/724-painweek2014acceptedabstracts.pdf.

3. Schwittay A et al., Effects of Tapentadol Prolonged Release (PR) Versus Oxycodone/Naloxone PR on Quality of Life and Function Measures in Patients with Severe Chronic Low Back Pain with a Neuropathic Pain Component. PAIN Week. Las Vegas, USA 2014. Abstract 123. Available at: http://conference.painweek.org/media/mediafile_attachments/04/724painweek2014acceptedabstracts.pdf.

4. Vondrackova D, et al. Analgesic efficacy and safety of oxycodone in combination with naloxone as prolonged release tablets in patients with moderate to severe chronic pain. J Pain 2008;9 (12):1144-54.

5. Lange B, et al. Efficacy and safety of tapentadol prolonged release for chronic osteoarthritis pain and low back pain. Adv Ther 2010;27(6):381-99.

6. Sánchez del Aguila MJ, et al. Practical considerations for the use of tapentadol prolonged release for the management of severe chronic pain. Clin Ther 2015;37(1):94-113.

7. Galvez R, et al. Tapentadol prolonged release versus strong opioids for severe, chronic low back pain: Results of an open-label, phase 3b study. Adv Ther 2013;30(3):229-59.

8. Cloutier $\mathrm{C}$, et al. Controlled-release oxycodone and naloxone in the treatment of chronic low back pain: A placebo-controlled, randomized study. Pain Res Manag 2013;18(2):75-82. 